This research was published in Nature. Mousavi, S. A., Chauvin, A., Pascaud, F., Kellenberger, S., and Farmer, E. E. (2013) GLUTAMATE RECEPTOR-LIKE genes mediate leaf-to-leaf wound signalling. Nature 500, 422-426, doi: 10.1038/nature12478

\title{
GLUTAMATE RECEPTOR-LIKE genes mediate leaf-to-leaf wound signalling
}

Seyed A. R. Mousavi ${ }^{\mathrm{a}}$, Adeline Chauvin ${ }^{\mathrm{b}}$, François Pascaud ${ }^{\mathrm{c}}$, Stephan Kellenberger ${ }^{\mathrm{c}}$ and Edward E. Farmer, ${ }^{\mathrm{a}, *}$

a. Department of Plant Molecular Biology, University of Lausanne, Biophore, CH-1015 Lausanne, Switzerland.

b. School of Pharmaceutical Sciences, University of Geneva, 30 quai Ernest-Ansermet, CH1211 Geneva 4, Switzerland

c. Department of Pharmacology and Toxicology, University of Lausanne, Rue du Bugnon 27, CH-1005 Lausanne, Switzerland

Address correspondence to: Edward E. Farmer, Department of Plant Molecular Biology, University of Lausanne, Biophore, CH-1015 Lausanne, Switzerland.

${ }^{*}$ To whom correspondence should be addressed. Tel.: 41-21-692-4228; Fax: 41-21-692-4195; E-mail: edward.farmer@unil.ch. 
Abstract

Wounded leaves communicate their damage status to one another through a poorly understood process of long distance signalling. This stimulates the distal production of jasmonates, potent regulators of defence responses. Using non-invasive electrodes we mapped surface potential changes in Arabidopsis thaliana after wounding a leaf and found that membrane depolarisations correlated with jasmonate signalling domains in undamaged leaves. Furthermore, current injection elicited jasmonoyl-isoleucine accumulation, resulting in an electrome enriched in transcripts encoding key jasmonate signalling regulators. Mutants in several clade 3 GLUTAMATE RECEPTOR-LIKE genes (GLRs 3.2, 3.3 and 3.6) attenuated wound-induced surface potential changes, and jasmonate-response gene expression in leaves distal to wounds was reduced in a glr3.3 glr3.6 double mutant. The work provides a genetic basis for investigating mechanisms of long distance defence signalling in plants and indicates that plant genes related to those important for synaptic activity in animals function in organ-to-organ wound signalling. 
Unlike plants, animals rely on rapid nervous systems to escape predation. A stationary fly that perceives danger takes less than $300 \mathrm{~ms}$ to take off, and this process requires complex wholebody coordination ${ }^{1}$. Nevertheless, this escape response is too slow if the fly lands on a Venus flytrap, a plant in which electrical signals initiate rapid trap closure ${ }^{2}$. While fast movements associated with insect capture are exceptional, slower herbivore-induced defence gene expression is widespread in plants and is coordinated between organs ${ }^{3}$. What, then, is the nature of the long distance signal that leads to defence responses throughout much of a plant body after wounding? Among the many scenarios proposed to explain the nature of systemic wound signals in plants ${ }^{4}$ is a role for electrical signalling ${ }^{5}$. However, this has not been substantiated and it is essential to identify genes that underlie this phenomenon.

Resistance to herbivores depends to a large extent on the production of potent lipids known as jasmonates ${ }^{6,7}$. Without the ability to produce or perceive these compounds, plants that normally resist attack become remarkably vulnerable to predation ${ }^{8}$. Both jasmonic acid (JA) and biologically active jasmonoyl-isoleucine (JA-Ile) ${ }^{9}$ accumulate within minutes in and near wounds and in undamaged distal tissues ${ }^{10-12}$. Similarly, when feeding on $A$. thaliana, the Egyptian cotton leafworm (Spodoptera littoralis) stimulates jasmonate-regulated transcript accumulation in tissues several centimetres from a wound ${ }^{13}$. When feeding on bean, these insect larvae are also known to provoke plasma membrane depolarisations that spread through entire wounded leaves ${ }^{14}$. Such damage-induced plasma membrane depolarisations are common in plants ${ }^{15,16}$ and are also produced after exposure of cells to damage-associated molecular patterns ${ }^{17}$, including peptide danger signals ${ }^{18}$ that have been associated with antipathogen defences ${ }^{19}$. Moreover, there is evidence that jasmonate synthesis can be stimulated by events that lead to plasma membrane depolarisation. For example, treatment of tomato cells with ionophores that cause plasma membrane depolarisation stimulated the expression of 
jasmonate-regulated genes ${ }^{20-21}$. Additionally, membrane depolarisations in potato preceeded increases in cytosolic $\mathrm{Ca}^{2+}$, and jasmonate accumulation was reduced when these $\mathrm{Ca}^{2+}$ transients were blocked ${ }^{22}$. Here, concentrating exclusively on the jasmonate defence pathway and using non-invasive surface electrodes ${ }^{23}$, we monitored changes in electrical activity due to ion fluxes in cell populations in wounded Arabidopsis plants. As summarised in Supplementary Fig. 1, we show that electrical signalling activates jasmonate biosynthesis in leaves distal to wounds and we identify genes involved in electrical signal propagation.

\section{Wound-induced surface potential changes}

To investigate patterns of electrical activity and gene expression in 5 week-old rosettes, individual leaves were numbered from oldest to youngest. Electrodes placed on leaf 8 at the midrib/petiole junction (e2 electrode position) and on the petiole (position e3) did not detect changes in electrical activity and such changes were not elicited by walking S. littoralis larvae (Fig. 1a and Supplementary Fig. 2a,b). When the larvae began to feed, wound-induced surface potential changes (WASPs) of variable amplitude, duration and complexity were observed (Supplementary video 1 and Supplementary Fig. 2c). Since insects release various chemical elicitors in addition to causing wounding ${ }^{3}$, we investigated the effects of mechanical wounding on electrical activity. Simply touching the leaf did not generate changes in surface potential, but wounding $40 \%$ of the leaf tip resulted in strong and reproducible surface potential changes (Fig. 1b). When recordings were extended, they often showed periodicity (Supplementary Fig. 3). We used three parameters to characterise these signals: latency (time from wounding to arrival at the amplitude midpoint), amplitude and duration (Fig. 1b). To gain more information on the spread of WASPs within a wounded leaf, four electrodes were placed on the leaf surface (Fig. 1a). After damage, WASPs were detected first at e1, then several seconds later at e2, and finally at e3. An electrode on the lamina also detected 
damage-elicited electrical activity and, in each case (Fig. 1c), the changes in amplitude were typically close to $-70 \mathrm{mV}$ (SupplementaryTable 1). The signals we measured had the same polarity as those produced after a chilling treatment known to cause plasma membrane depolarisation $^{24,25}$. Therefore WASPs in leaf 8 were due to plasma membrane depolarisation (Supplementary Fig. 4). The WASPs detected on WT plants were indistinguishable to those on wounded plants that lacked the ability to synthesize jasmonates (Supplementary Fig. 5). This suggests that the mechanism that produces WASPs is upstream or independent of jasmonate synthesis.

\section{WASP territories and speeds}

Signals generated by wounding leaf tips first move towards the centre of the rosette and then disperse away from the apex into a restricted number of distal leaves to initiate distal JA accumulation and signalling ${ }^{11}$. In order to map the spatial distribution of WASPs in the rosette after wounding leaf 8 we placed electrodes in the e 3 position of leaves 5 through 18 . Leaves $5,11,13$ and 16 showed responses similar to those in the wounded leaf (Fig. 1d, Supplementary Table 2). For example, after wounding leaf 8, a WASP with a duration of $78 \pm 20 \mathrm{~s}$ and a peak amplitude of $-51 \pm 9 \mathrm{mV}$ was reached in leaf 13 after a latency of $66 \pm 13$ $\mathrm{s}(\mathrm{n}=61$ plants). Other leaves $(7,9,10,12,14,15,17$ and 18$)$ showed small positive surface potential changes. For example, leaf 9 showed a $20 \pm 5 \mathrm{mV}$ change in surface potential with a latency of $54 \pm 12 \mathrm{~s}$ ( $\mathrm{n}=46$ plants). Most of these observations fit a developmental pattern: In adult-phase Arabidopsis rosettes, leaf ' $n$ ' shares direct vascular connections to leaves $n \pm 5$ and $\mathrm{n} \pm 8$. Thus the wounded leaf 8 is connected to leaves 13 and 16 , these connections being termed parastichies ${ }^{26}$. However, in addition to leaves 13 and 16, leaves 5 and 11 also showed strong negative surface potential changes after wounding leaf 8 . These leaves are $n \pm 3$ relative to the wounded leaf 8 and may represent contact parastichies formed by proximal but 
unconnected vasculature ${ }^{26}$. Additionally, we recorded changes in surface potentials in the n-2 leaf (leaf 6) that were similar to those in wounded leaf 8 in $63 \%$ recordings. The remaining recordings from leaf 6 (Fig. 1d), resembled traces from leaves such as leaf 9. We termed leaf 6 a variable leaf.

Quantitative electrophysiological data (Supplementary Table 2) was then compared with transcript levels for JASMONATE-ZIM DOMAIN 10 (JAZ10), a robust marker for activity of the jasmonate pathway ${ }^{27}$. One $\mathrm{h}$ after wounding leaf 8 we detected $>100$ fold increases in $J A Z 10$ transcript levels in leaves 5, 8,11, 13 and 16 (Fig. 1e). JAZ10 transcript induction in leaf 6 was variable. Heat maps from quantitative data show that $J A Z 10$ expression at $1 \mathrm{~h}$ postwounding and WASP durations covered identical territories, spanning $137^{\circ}$ of the rosette when variable leaf 6 (n-2) is included (Fig. 1f). To test whether WASP propagation occurred from young to older leaves we wounded leaf 13 and monitored events in leaf 8 . Like signalling from older to younger leaves we observed a correlation in the spatial pattern of WASP production and JAZ10 expression (Supplementary Fig. 6).

We next examined the speed at which electrical signals moved within wounded leaves and from leaf to leaf. Replicated measurements (Supplementary Table 1 and Supplementary Fig. 7) indicated a range of speeds from $2.6 \pm 0.6 \mathrm{~cm} \mathrm{~min}^{-1}$ between the wound and an electrode placed on the lamina, to up to $9 \mathrm{~cm} \mathrm{~min}^{-1}$ between electrodes placed on the midribs of the wounded leaf itself or at intervals along the midrib on leaf 13. These similar apparent velocities for surface potential changes in the midribs of wounded leaves and distal leaves suggest that related mechanisms control electrical signalling in these leaves. However, signals from the wounded leaf appeared to slow to $5.4 \pm 1.5 \mathrm{~cm} \mathrm{~min}^{-1}$ at the center of the plant prior to accelerating again in the distal leaf, bringing the average signal speed from wounded leaf 8 to 
receiver leaf 13 to $5.8 \pm 1.1 \mathrm{~cm} \mathrm{~min}^{-1}(\mathrm{n}=13)$. This overall velocity estimate is concordant with recent estimates of signal speeds based on JA accumulation in leaf 13 of Arabidopsis after wounding leaf $8^{\text {(ref. 28) }}$, and with self-propagating electrical activity elicited by wounding bean or barley leaves ${ }^{29}$. To investigate whether the long distance signals that activate jasmonate responses travel at similar speeds to WASP changes, electrodes were placed in positions e2 and $\mathrm{e} 3$ on leaf 8 and this leaf was then wounded. When the wounded leaf 8 was severed between e 2 and e 3 after a signal had reached e 3 we detected induced $J A Z 10$ expression in leaf 13. However, JAZ10 induction was not observed if we cut the wounded leaf as the WASP arrived at e2 but before it reached e3 (Supplementary Fig. 8). We conclude that the signal that strongly activated $J A Z 10$ expression travelled at a similar speed to electrical events elicited by wounding.

\section{Current injection and the Arabidopsis electrome}

To test for a direct link between the jasmonate pathway and electrical activity we implanted platinum (Pt) wires into the petioles of leaf 8 (Fig. 2a), injected current, and monitored the induction of WASPs in the lamina of this leaf. By structuring the input current appropriately (40 $\mu$ A for $10 \mathrm{~s}$; Supplementary Table 3) we were able to induce surface potential changes distal to the site of injection (Fig. 2b) without causing detectable cell damage other than that due to Pt wire implantation in the petiole, a region that was removed prior to analysing the lamina (Supplementary Fig. 9). The signals generated in response to current injection (ci) had a mean duration of $59 \pm 25 \mathrm{~s}$ (Supplementary Table 4), similar to the durations of WASPs in leaf 13 when leaf 8 was wounded. From these data we estimated the apparent velocity of the surface potential change resulting from ci to be $6.4 \pm 1.9 \mathrm{~cm} \mathrm{~min}^{-1}$. This was close to an average action potential velocity of $7 \mathrm{~cm} \mathrm{~min}^{-1}$ that has been observed after ci in Arabidopsis $^{30}$. Furthermore, ci has been shown to stimulate the accumulation of JA in 
tomato $^{31}$. We therefore measured the levels of both JA and JA-Ile at $20 \mathrm{~min}$ and $1 \mathrm{~h}$ after ci in leaf 8. Current injection stimulated JA and JA-Ile accumulation at both timepoints (Fig. 2c and d; Supplementary Fig. 10).

To confirm that ci could induce jasmonate signalling the expression of two jasmonateresponsive genes, the regulatory gene JAZ10 and VEGETATVE STORAGE PROTEIN 2 (VSP2), an anti-insect defence gene ${ }^{32}$, was monitored. Transcripts for both genes were upregulated strongly in response to ci (Fig. 2e and f). When current was injected into the coronatine-insensitive 1-1 (coil-1) ${ }^{33}$, a mutant lacking the functional jasmonate receptor, we recorded WASPs similar to those in the current injected WT (Supplementary Fig. 11a). However, we were unable to induce $J A Z 10$ expression in these plants (Supplementary Fig. 11b). The canonical jasmonate signal pathway ${ }^{6}$ is therefore required for expression of $J A Z 10$ after ci. Consistent with the detection of WASPs over entire leaf surfaces, plants expressing a wound-inducible $V S P 2$ reporter (Fig. 2g) also responded to ci throughout the lamina (Fig. 2h).

To find out if other jasmonate-regulated genes were activated by ci we performed whole transcriptome analysis starting with the electrome of current-injected leaf 8 . This revealed that 313 genes were $>2$-fold upregulated (Supplementary Table 5, Fig. 3). We then generated a second dataset from leaf 13 one h after wounding leaf 8 . Finally, we compared these results with data produced independently from wounded 18 day-old plants ${ }^{34}$. Comparison of the datasets showed that $94 \%$ of the ci-upregulated genes were also upregulated in leaf 13 of plants wounded on leaf $8.70 \%$ of ci-induced transcripts were upregulated in both leaf wounding datasets. Strikingly, among these were 9 of the 12 Arabidopsis JAZ genes (Supplementary Table 6 and Fig. 3). These genes are critical regulators of jasmonate signalling ${ }^{4,6,27}$. It is known that more transcripts are upregulated than are downregulated in 
response to wounding ${ }^{13,27,34}$. Consistent with this, the levels of only 66 transcripts decreased in response to ci. Of these transcripts, $47 \%$ were also downregulated in one or both wounding experiments (Supplementary Table 7 and Supplementary Fig. 12). Clearly, ci does not affect the expression of all wound-regulated genes and this is consistent with the fact that several signal pathways operate in damaged Arabidopsis ${ }^{4,27,35,36}$. One such pathway depends on NADPH oxidase D (RBOHD) to transmit reactive oxygen species (ROS)-dependent long distance signals at speeds similar to the WASP velocities we describe ${ }^{36}$. Although inhibitors of this ROS propagation pathway ${ }^{36}$ did not abolish WASPs (Supplementary Fig. 13a-c), we found that one of them, diphenyleneiodonium (DPI), reduced WASP duration in leaf 13 by a factor of 2. However, DPI did not inhibit distal JAZ10 expression (Supplementary Fig. 13d) and wound-induced $J A Z 10$ expression was not reduced relative to the WT in rbohD mutants (Supplementary Fig. 14) in which WASP production was similar to that in the WT (Supplementary Table 9). In conclusion, the propagating signal leading to distal JAZ10 expression is likely to be RBOHD-independent.

\section{GLR genes mediate long distance wound signalling}

There is growing evidence that genes encoding various ion channels and pumps can affect jasmonate signalling $^{37-39}$. For example, the overexpression of a GLUTAMATE RECEPTORLIKE (GLR) gene from radish stimulated the expression of jasmonate-regulated genes including VSP1 in Arabidopsis ${ }^{40}$. Using a reverse genetic approach based on monitoring electrical activity after wounding leaf 8, we screened homozygous Arabidopsis mutants in putative pumps and ion channels including GLRs (Supplementary Tables 8 and 9). Mutations in 4 genes: GLR3.1, GLR3.2 (two alleles), GLR3.3 (two alleles) and GLR3.6 (two alleles), all of which reduced GLR expression (Supplementary Fig. 15), caused reduced durations of 
surface potential changes either in leaf 8 , leaf 13, or both (Supplementary Table 9 and Fig. 4a). Two of the $g l r$ mutations, one that reduced WASP duration in leaf $8(g l r 3.3 a)$, and one that reduced WASP duration in leaf $13(\mathrm{glr} 3.6 \mathrm{a})$, were combined to produce a double mutant glr3.3a glr3.6a. The double mutant, an a second related double mutant showed reduced electrical activity in wounded leaf 8 but, unlike the single mutants, changes in surface potential were no longer detectable in leaf 13 when leaf 8 was wounded (Fig. 4a and Supplementary Table 9). Additionally, we were unable to stimulate electrical activity after current injection in the double mutant (Fig. 4b) and the elevation of JAZ10 transcript levels seen in leaf 8 of the WT after ci was almost abolished (Fig. 4c). Finally, although wounding caused elevated $J A Z 10$ transcript levels in wounded leaf 8 of the WT, in the glr single mutants, and in the glr3.3 glr3.6 double mutant, JAZ10 expression was reduced in distal leaf 13 of both glr single mutants (Fig. 4d) as well as in the second allele of glr3.3

(Supplementary Fig. 16). A stronger reduction in wound-induced JAZ10 transcript level relative to the WT was seen in distal leaf 13 of the glr3.3a glr3.6a double mutant (Fig. 4d).

\section{Discussion}

We have identified genes involved in the propagation of electrical activity leading to defence gene expression. The findings are consistent with a previous report implicating electrical signalling in the distal activation of proteinase inhibitor gene expression in tomato seedlings. ${ }^{5}$ The GLR genes we studied encode putative cation channels, and GLR3.3 functions in agoniststimulated plasma membrane depolarisation ${ }^{41,42}$. This gene ${ }^{41}$, as well as several GLRS expressed in pollen ${ }^{43}$, can control cytosolic $\mathrm{Ca}^{2+}$ influxes, and GLRs have also been implicated in mediating calcium influxes in response to the perception of microbe-associated molecular patterns ${ }^{44}$. Our results show that GLRs control the distal wound-stimulated expression of several key jasmonate-inducible regulators of jasmonate signalling ( $J A Z$ genes). 
Finally, GLRs are related to ionotropic glutamate receptors (iGluRs) that are important for fast excitatory synaptic transmission in the vertebrate nervous system ${ }^{45}$. They and their plant relatives may control signalling mechanisms that existed prior to the divergence of animals and plants ${ }^{46}$. If so, a deeply conserved function for these genes might be to link damage perception to distal protective responses.

\section{METHODS SUMMARY}

Arabidopsis thaliana accession Col-0 and T-DNA insertion lines were obtained from the Nottingham Arabidopsis Stock Centre (NASC). Their homozygosity was confirmed prior to all experiments. Surface potentials were recorded with silver/silver chloride electrodes placed in $10 \mu \mathrm{l}$ of $10 \mathrm{mM} \mathrm{KCl}$ in $0.5 \%(\mathrm{w} / \mathrm{v})$ agar. The ground electrode was placed in the soil. Current injection was carried out via two platinum wires that were inserted into the leaf one day prior to the experiment. Quantitative RT-PCR for JAZ10 (At5g13220) and VSP2 (At5g24770) was done from reverse-transcribed RNA by SYBR Green assays and standardized to ubiquitin-conjugating enzyme (UBC21, At5g25760). For transcriptome analysis, amplified RNA was hybridized to Affymetrix ATH1 arrays. Probe sets showing at least a two-fold change and a false discovery rate $<0.05$ were considered significant ${ }^{47}$. Jasmonates, extracted according to Glauser et al., ${ }^{11}$ were separated by high-performance liquid chromatography (Phenomenex Kinetex (Torrance, CA) 2.6 mm C18 $100 \AA$ column) and quantified by electrospray ionization mass spectrometry in the multiple reaction monitoring mode.

Full Methods and any associated references are available in the online version of the full paper.

Supplementary information is available in the online version of the paper. 


\section{Acknowledgements}

This work was supported by Swiss NSF grants 3100A0-122441 and 31003A-138235 (to EEF), SystemsX.ch (to EEF) and a Faculty of Biology and Medecine Interdisciplinary grant (to SK and EEF). We thank I. Acosta and D. Gasperini and all other members of the Farmer lab for critical comments, S. Stolz and A. Chételat (University of Lausanne) for excellent technical help, M. Blanchard (University of Lausanne) for help with electrophysiology, P. Schweizer and P. Reymond (University of Lausanne) for insect larvae, and M. Shakhsi-Niaei (University of Bern) for help with transcriptome analyses that were conducted in the Lausanne Genomic Technologies Facility. We thank Y. Lee (University of Lausanne) and F. Mauch (University of Fribourg) for $r b o h D$ seeds and J-L. Wolfender (University of Geneva) for support with jasmonate analyses. R. Benton, C. Fankhauser, N. Geldner, C. Hardtke and Y. Poirier (University of Lausanne) are thanked for valuable comments.

Author contributions SARM, AC, FP and SK performed experiments; EEF, SARM and SK conceived experiments; EEF and SK wrote the manuscript.

Author information Gene expression data are available in the GEO database under accession number GSE41779. The authors declare no competing financial interests. Correspondence and requests for materials should be addressed to E.E.F (edward.farmer@unil.ch). 


\section{FIGURE LEGENDS}

Figure 1: WASPS and $J A Z 10$ expression map to identical spatial domains. a. Experimental design for detecting surface potential changes on leaves. a. The approximate size of leaf 8 from 5 week-old in Arabidopsis plants was $5 \mathrm{~cm}$ in length (including petiole) and $1.5 \mathrm{~cm}$ in width. Measuring electrodes were placed at $1 \mathrm{~cm}$ intervals on this leaf: midrib (e1), petiole/midrib junction (e2), petiole (e3). The lamina electrode (eL) was $3 \mathrm{~mm}$ from e1. The apical part of the leaf was wounded with forceps. b. Three distinct variables, latency, duration and amplitude of WASPs were analysed. Duration is the time between amplitude change midpoints. Latency (Lat.) is period between wounding and WASP detection. Amplitude was relative to the baseline prior to wounding. The signal in the wounded leaf (leaf 8 , recorded at e3) typically did not recover to baseline during recording (unfilled arrowhead). Time of wounding is indicated with a filled arrowhead. c. Examples of typical surface potential 
changes recorded on leaf 8 . Arrowheads indicate when the leaf was either touched or wounded. d. Representative WASPs generated on distal leaves after wounding leaf 8 . The data are representative of 10-61 independent replicates. Two types of surface potential change were observed on leaf 6 . The solid line shows a representative trace from $63 \%(n=19)$ of events and the dashed line indicates an opposite polarity change in surface potential seen in $37 \%$ of cases. e. Levels of $J A Z 10( \pm \mathrm{SD})$ transcript relative to unwounded leaf 8 without wounding (upper panel) and $1 \mathrm{~h}$ after mechanical wounding of leaf 8 (lower panel). $* * *=$ $P<0.001$. For leaf $6 P>0.05$. Numbers within bars show the product of amplitude and duration (mV.s) for leaves that displayed depolarisations. f. Heat maps representing the level of JAZ10 transcript induction $1 \mathrm{~h}$ after wounding leaf 8 and the duration of surface potential changes produced after wounding leaf 8 . Only leaves that were investigated are indicated. Data for $J A Z 10$ levels are from panel $\mathrm{E}$ in this figure. WASP durations are from Table $\mathrm{S} 2 . \mathrm{v}=$ variable leaf.

Figure 2: Current injection (ci) induces jasmonate accumulation, gene expression and surface potentials. a. Placement of Pt wires, proximal electrode (eP), distal electrode $(\mathrm{eD})$ and laminar electrode $(\mathrm{eL})$ for ci experiments. The leaf blade to the left of the dashed line was used for transcript measurements and quantification of jasmonate levels. b. Surface potential generation following ci $(40 \mu \mathrm{A}$ for $10 \mathrm{~s})$. Art = artefacts recorded in the leaf during ci. $(10 \mathrm{~s}$, indicated with a bar). Note that the signal amplitude at eP reaches a maximum before that at $\mathrm{eD}$ and eL. c. Levels of JA ( \pm SD) $20 \mathrm{~min}$ and $1 \mathrm{~h}$ after ci $(40 \mu \mathrm{A}, 10 \mathrm{~s})$. d. Levels of JA-Ile ( \pm SD) 20 min and $1 \mathrm{~h}$ after ci. Limits of quantitation (LOQs) are indicated with dashed lines. For JA and JA-Ile levels after wounding in control experiments see Fig. S9. e. Levels of $J A Z 10$ transcripts $( \pm \mathrm{SD})$ in leaves $1 \mathrm{~h}$ after wounding or ci. f. Levels of $V S P 2$ transcripts ( \pm SD) in leaves $4 \mathrm{~h}$ after wounding or ci. Control leaves (no ci) carried Pt wire implants but 
were not subjected to ci. $\mathrm{U}=$ unwounded plants. $\mathrm{W}=$ wounded. $* * *=P<0.001$. In e and $\mathrm{f}$ transcript levels were normalised to the unwounded control. g. Expression pattern of a VSP2:GUSPlus reporter line in leaf 8 from an unwounded plant, and in leaf $84 \mathrm{~h}$ after wounding. h. VSP2:GUSPlus activity in leaf 8 of control plants (no ci) and $4 \mathrm{~h}$ after ci.

Figure 3. Current injection and wounding stimulate the expression of a common $J A Z$ geneenriched subset of genes. Venn diagram for the number of transcripts upregulated $>2$-fold compared to unstimulated plants $(P \leq 0.05)$ for current-injected leaf $8(40 \mu \mathrm{A}, 10 \mathrm{~s}$; this study), for leaf 13 of plants that had been wounded on leaf 8 (this study), and for wounded Arabidopsis rosettes ${ }^{34}$.Numbers in parentheses are upregulated $J A Z$ genes.

Figure 4: $g l r$ mutants reduce the duration of WASPs, and responses to current injection. a. Typical WASPs from the wounded leaf 8 of the WT, glr3.3a (At1g42540, SALK line 099757), glr3.6a (At3g51480, SALK line 091801) and the double mutant glr3.3a glr3.6a and surface potential recordings from leaf 13 of the same genotypes wounded on leaf 8 . The dashed line indicates WASP duration. b. Lack of surface potential generation in leaf 8 of the glr3.3a glr3.6a double mutant after current injection. c. $J A Z 10$ expression ( \pm SD) after current injection (ci) in the WT and the glr3.3a glr3.6a double mutant. d. JAZ10 expression ( \pm SD) after wounding leaf 8 in the WT, glr single mutants, and the glr3.3a glr3.6a double mutant. For c and d RNA samples were collected $1 \mathrm{~h}$ after stimulation and levels of JAZ10 transcripts were normalised to the resting WT control. $* *=P<0.01, * * *=P<0.001$.

\section{References:}

1 Card, G. \& Dickinson, M. H. Visually mediated motor planning in the escape response of Drosophila. Curr. Biol. 18, 1300-1307 (2008). 
Escalante-Pérez, M. et al. A special pair of phytohormones controls excitability, slow closure, and external stomach formation in the Venus flytrap. Proc. Natl. Acad. Sci. 108, 15492-15497 (2011).

3 Walters, D. R. Plant Defense. Blackwell Publishing Ltd (2011).

4 Koo, A. J. K. \& Howe, G. A. The wound hormone jasmonate. Phytochemistry 70, 1571-1580 (2009).

5 Wildon, D. C. et al. Electrical signaling and systemic proteinase-inhibitor induction in the wounded plant. Nature 360, 62-65 (1992).

6 Browse, J. Jasmonate passes muster: A receptor and targets for the defense hormone. Annu. Rev. Plant Biol. 60, 183-205 (2009).

7 Wasternack, C. \& Hause, B. Jasmonates: biosynthesis, perception, signal transduction and action in plant stress response, growth and development. An update to the 2007 review in Annals of Botany. Ann. Bot. in press (2013). doi:10.1093/aob/mct067 Howe, G. A. \& Jander, G. Plant immunity to insect herbivores. Annu. Rev. Plant Biol. 59, 41-66 (2008).

9 Fonseca, S. et al. (+)-7-iso-Jasmonoyl-L-isoleucine is the endogenous bioactive jasmonate. Nat Chem Biol 5, 344-350 (2009).

10 Glauser, G. et al. Spatial and temporal dynamics of jasmonate synthesis and accumulation in Arabidopsis in response to wounding. J. Biol. Chem. 283, 1640016407 (2008).

11 Glauser, G. et al. Velocity estimates for signal propagation leading to systemic jasmonic acid accumulation in wounded Arabidopsis. J. Biol. Chem. 284, 3450634513 (2009).

12 Koo, A. J. K., Gao, X., Jones, A. D. \& Howe, G. A. A rapid wound signal activates the systemic synthesis of bioactive jasmonates in Arabidopsis. Plant J. 59, 974-986 (2009).

13 Reymond, P. et al. A conserved transcript pattern in response to a specialist and a generalist herbivore. Plant Cell 16, 3132-3147 (2004).

14 Maffei, M., Bossi, S., Spiteller, D., Mithöfer, A. \& Boland, W. Effects of feeding Spodoptera littoralis on Lima bean leaves. I. Membrane potentials, intracellular calcium variations, oral secretions, and regurgitate components. Plant Physiol. 134, 1752-1762 (2004).

15 Fromm, J. \& Lautner, S. Electrical signals and their physiological significance in plants. Plant Cell Environ. 30, 249-257 (2007).

16 Stahlberg, R., Cleland, R. E. \& Volkenburgh, E. V. in Communication in plants (eds F. Baluška, S. Mancuso, \& D. Volkmann) 291-308 (Springer-Verlag, 2006 ).

17 Boller, T. \& Felix, G. A Renaissance of elicitors: perception of microbe-associated molecular patterns and danger signals by pattern-recognition receptors. Annu. Rev. Plant Biol. 60, 379-406 (2009).

18 Krol, E. et al. Perception of the Arabidopsis danger signal peptide 1 involves the pattern recognition receptor AtPEPR1 and its close homologue AtPEPR2. J. Biol. Chem. 285, 13471-13479 (2010).

19 Huffaker, A., Pearce, G. \& Ryan, C. A. An endogenous peptide signal in Arabidopsis activates components of the innate immune response. Proc. Natl. Acad. Sci. 103, 10098-10103 (2006).

20 Schaller, A. \& Oecking, C. Modulation of plasma membrane $\mathrm{H}^{+}$-ATPase activity differentially activates wound and pathogen defense responses in tomato plants. Plant Cell 11, 263-272 (1999).

21 Schaller, A. \& Frasson, D. Induction of wound response gene expression in tomato leaves by ionophores. Planta 212, 431-435 (2001). 
22 Fisahn, J., Herde, O., Willmitzer, L. \& Peña-Cortés, H. Analysis of the transient increase in cytosolic $\mathrm{Ca}^{2+}$ during the action potential of higher plants with high temporal resolution: requirement of $\mathrm{Ca}^{2+}$ transients for induction of jasmonic acid biosynthesis and PINII gene expression. Plant Cell Physiol. 45, 456-459 (2004).

23 Stahlberg, R. \& Cosgrove, D. J. Comparison of Electric and Growth-Responses to Excision in Cucumber and Pea-Seedlings .1. Short-Distance Effects Are a Result of Wounding. Plant Cell Environ. 17, 1143-1151 (1994).

24 Carpaneto, A. et al. Cold Transiently Activates Calcium-Permeable Channels in Arabidopsis Mesophyll Cells. Plant Physiol. 143 487-494 (2007).

25 Minorsky, P. V. Temperature sensing by plants: a review and hypothesis. Plant Cell Environ. 12, 119-135 (1989).

26 Dengler, N. G. The shoot apical meristem and development of vascular architecture. Can. J. Bot. 84, 1660-1671 (2006).

27 Yan, Y. et al. A downstream mediator in the growth repression limb of the jasmonate pathway. Plant Cell 19, 2470-2483 (2007).

28 Chauvin, A., Caldelari, D., Wolfender, J-L. \& Farmer, E.E. Four 13-lipoxygenases contribute to rapid jasmonate synthesis in wounded Arabidopsis leaves: a role for LOX6 in responses to long distance wound signals. New Phytol. 197, 566-575 (2013).

29 Zimmermann, M. R., Maischak, H., Mithofer, A., Boland, W. \& Felle, H. H. System potentials, a novel electrical long-distance apoplastic signal in plants, induced by wounding. Plant Physiol. 149, 1593-1600 (2009).

30 Favre, P. \& Agosti, R. D. Voltage-dependent action potentials in Arabidopsis thaliana. Physiol. Plant. 131, 263-272 (2007).

31 Herde, O. et al. Localized wounding by heat initiates the accumulation of proteinase inhibitor II in abscisic acid-deficient plants by triggering jasmonic acid biosynthesis. Plant Physiol. 112, 853-860 (1996).

32 Liu, Y. et al. Arabidopsis vegetative storage protein is an anti-insect acid phosphatase. Plant Physiol. 139, 1545-1556 (2005).

33 Xie, D.-X., Feys, B. F., James, S., Nieto-Rostro, M. \& Turner, J. G. COI1: An Arabidopsis gene required for jasmonate-regulated defense and fertility. Science 280, 1091-1094 (1998).

34 Kilian, J. et al. The AtGenExpress global stress expression data set: protocols, evaluation and model data analysis of UV-B light, drought and cold stress responses. Plant J. 50, 347-363 (2007).

35 Walley, J. W. et al. Mechanical stress induces biotic and abiotic stress responses via a novel cis-Element. PLoS Genet 3, 1800-1812 (2007).

36 Miller, G. et al. The plant NADPH oxidase RBOHD mediates rapid systemic signaling in response to diverse stimuli. Sci. Signal. 2, ra45-, doi: 10.1126/scisignal.2000448 (2009).

37 Brüx, A. et al. Reduced V-ATPase activity in the trans-Golgi network causes oxylipin-dependent hypocotyl growth Inhibition in Arabidopsis. Plant Cell 20, 10881100 (2008).

38 Bonaventure, G. et al. A gain-of-function allele of TPC1 activates oxylipin biogenesis after leaf wounding in Arabidopsis. Plant J. 49, 889-898 (2007).

39 Beyhl, D. et al. The fou 2 mutation in the major vacuolar cation channel TPC1 confers tolerance to inhibitory luminal calcium. Plant J. 58, 715-723 (2009).

40 Kang, S. et al. Overexpression in Arabidopsis of a plasma membrane-targeting glutamate receptor from small radish increases glutamate-mediated $\mathrm{Ca}^{2+}$ influx and delays fungal infection. Mol. Cell. 21, 418-427 (2006). 
41 Qi, Z., Stephens, N. R. \& Spalding, E. P. Calcium entry mediated by GLR3.3, an Arabidopsis glutamate receptor with a broad agonist profile. Plant Physiol. 142, 963971 (2006).

42 Stephens, N. R., Qi, Z. \& Spalding, E. P. Glutamate receptor subtypes evidenced by differences in desensitization and dependence on the GLR3.3 and GLR3.4 genes. Plant Physiol. 146, 529-538 (2008).

43 Michard, E. et al. Glutamate receptor-like genes form $\mathrm{Ca}^{2+}$ channels in pollen tubes and are regulated by pistil D-serine. Science 332, 434-437 (2011).

44 Kwaaitaal, M., Huisman, R., Maintz, J., Reinstädler, A. \& Panstruga, R. Ionotropic glutamate receptor (iGluR)-like channels mediate MAMP-induced calcium influx in Arabidopsis thaliana. Biochem. J. 440, 355-365 (2011).

45 Traynelis, S. F. et al. Glutamate receptor ion channels: structure, regulation, and function. Pharmacol. Rev. 62, 405-496 (2010).

46 Chiu, J. C. et al. Phylogenetic and expression analysis of the glutamate-receptor-like gene family in Arabidopsis thaliana. Mol. Biol. Evol. 19, 1066-1082 (2002).

47 Benjamini, Y. \& Hochberg, Y. Controlling the false discovery rate: a practical and powerful approach to multiple testing. J. R. Stat. Soc. Ser. B Methodol. 57, 289-300 (1995).

\section{Supplementary methods}

\section{Plant material and growth conditions and bioassays}


Arabidopsis thaliana (Columbia), were soil-grown (one seed per $7 \mathrm{~cm}$ diameter pot) for 5 to 6 weeks with $10 \mathrm{~h}$ light $\left(100 \mu \mathrm{E} \mathrm{sec} \mathrm{se}^{-1}\right), 70 \%$ humidity; day $22^{\circ} \mathrm{C}$, night $18^{\circ} \mathrm{C}$. One single wounding experiment was carried out per plant. Wounds were inflicted with plastic nonlocking thumb forceps that had flat, $4 \mathrm{~mm}$-wide ridged tips. The space between each ridge was $1 \mathrm{~mm}$ with an inter-ridge depth of $0.6 \mathrm{~mm}$. Wounds were inflicted with these ridges parallel to the long axis of the leaf. The first wound was made at the leaf tip and the second wound was made so that it abutted the first, and so on until c. $40 \%$ of the leaf was wounded. The wounding procedure takes about $10 \mathrm{~s}$ to complete. Prior to electrophysiology experiments plants were moved into a Faraday cage under the same light conditions. Spodoptera littoralis (4th instar larvae, from P. Reymond, University of Lausanne) were placed on plants or the apical parts of the leaves (40\% leaf area) were crushed with plastic forceps. A transparent plastic support was used to stabilize the wounded leaf during the experiments.

\section{Surface potential recordings and current injection}

For surface potential recordings, silver electrodes $0.5 \mathrm{~mm}$ in diameter (World Precision Instruments, Sarasota, FL) were chloridized with $\mathrm{HCl}(0.1 \mathrm{M})$, stored at room temperature and rechloridized after several uses. Experiments were conducted in a controlled environment room without changing the growth conditions. Two 2-channel amplifiers (FD 223 and Duo 773, World Precision Instruments, Sarasota, FL) were simultaneously used to record the surface potential at 4 positions. The electrode-leaf interface was a drop $(10 \mu \mathrm{l})$ of $10 \mathrm{mM} \mathrm{KCl}$ in $0.5 \%(\mathrm{w} / \mathrm{v})$ agar placed so that the Ag electrode did not contact and damage the cuticle. The interelectrode distance was the distance between the nearest edges of these agar droplets. The ground electrode was placed in the soil. The procedure for data quantitation is shown in Fig. $1 \mathrm{~b}$ in the main text. For experiments on interrupting signals, ceramic scissors (CS-250 Kyocera, Kyoto, Japan) were used. For current injection two platinum wire electrodes 
(Advent Research Materials, Oxford, UK), $0.1 \mathrm{~mm}$ diameter were inserted in the midrib $1 \mathrm{~cm}$ apart so that the end of the wire was visible from the abaxial leaf side but did not make contact with the soil (Fig. 2a). After insertion of the Pt wires the plants were rested for $24 \mathrm{~h}$ prior to experiments. For current injection the two Pt wires were connected to a homemade current source that was controlled by the acquisition program. Current was injected between the two Pt wires and the wire closest to the leaf lamina served as the positive electrode. A current of $40 \mu \mathrm{A}$ was injected during $10 \mathrm{~s}$. Control measurements indicated that during the injection of $40 \mu \mathrm{A}$ the voltage difference between the two platinum electrodes was $12.7+/$ $0.9 \mathrm{~V}(\mathrm{n}=9)$. In the current injection experiments, surface potentials were recorded as described above. In the combined experiments, the Chartmaster program via the InstruTECH LIH $8+8$ interface (HEKA Electronic, Lambrecht-Pfalz, Germany) was used to record the induced surface potential changes and to control the time and duration of current injection. In the experiments without current injection, surface potentials were recorded with Datatrax2 software via the LabTrax-4/16 interface (World Precisions Instruments, Sarasota, FL). The sampling interval was $10 \mathrm{~ms}$. Control plants were implanted with Pt wires in all current injection experiments. Trypan blue staining ${ }^{48}$ was used to assess the extent of damage caused by implanting Pt wires in petioles. The wires were implanted $24 \mathrm{~h}$ prior to staining. For $\beta$ glucuronidase (GUS) reporter plants the current injected leaf and the cognate control leaf were harvested for GUS staining for $15 \mathrm{~h}$ at $37{ }^{\circ} \mathrm{C}$. The tissue was destained in $70 \%$ ethanol ${ }^{49}$. Leaves (excluding cotyledons) were numbered from old to young.

\section{Ion leakage}

To investigate the effects of current injection on ion leakage, Pt current injection wires were inserted into petioles and the plants were left overnight prior to current injection ( $40 \mu \mathrm{A}, 10 \mathrm{~s})$. 
Three surface electrodes (eP, eD and eL, placed in $0.5 \% \mathrm{w} / \mathrm{v}$ agar droplets $(10 \mu \mathrm{l})$ containing $10 \mathrm{mM} \mathrm{KCl}$ were placed on the lamina (see Fig. 2a ) and used to monitor current-induced depolarisations. As controls, another set of plants were implanted with Pt wires and surface electrodes but not subjected to current injection. After current injection plants were incubated in the light for $1 \mathrm{~h}$ then the current-injected leaves and control leaves were cut off at the base of petiole. Before conductivity measurements the leaf surfaces were briefly washed with water to remove the agar droplets and petioles were attached so that only the part of the lamina that is harvested for JAZ10 measurements (see Fig. 2a) came into contact with deionised water $(25 \mathrm{ml} ; 3$ leaves per measurement). After 20 min gentle agitation the leaves were removed and the conductivity of the water was measured at $22^{\circ} \mathrm{C}$ with a Hanna Instruments EC215 conductivity metre (Distrelec, Zurich, Switzerland). Positive controls were leaves infiltrated on either side of the midrib of the abaxial lamina, each time with $10 \mu \mathrm{l}$ of $1 \%(\mathrm{v} / \mathrm{v})$ Triton $\mathrm{X}-100,1 \mathrm{~h}$ prior to ion leakage analysis. Negative controls were untreated leaves and an additional control for Triton X-100 infiltrations was water into which $20 \mu \mathrm{l}$ of the $1 \%$ Triton-X-100 solution was added.

\section{Pharmacological treatments}

$\mathrm{LaCl}_{3}$, catalase and diphenyleneiodonium (DPI) were from Sigma. Solutions were made in water; the DPI solution contained in addition $1 \%(\mathrm{v} / \mathrm{v})$ dimethyl sulphoxide (DMSO). The abaxial surface of each side of the main vein of leaf 8 was infiltrated with the inhibitor $(10 \mu 1)$ in the part that was later wounded. 25-30 min after infiltration leaf 8 was wounded and WASPs were recorded on leaf 13. Control experiments were carried out in exactly the same way with infiltration solutions that contained only the corresponding solvent. A similar experimental design was used for $J A Z 10$ expression analyses. Compounds were infiltrated 
into leaf 8 (two $10 \mu$ infiltrations into the abaxial lamina). 30 min after infiltration leaf 8 was wounded and RNA was harvested $1 \mathrm{~h}$ later.

\section{Quantitative PCR}

Total RNA was extracted with an RNeasy Plant Mini Kit (Qiagene, Germany) or with DNAfree RNA isolation protocols ${ }^{50}$. Total RNA $(1 \mu \mathrm{g})$ was copied into cDNA with M-MLV Reverse Transcriptase, RNase H Minus, Point Mutant first strand synthesis system (Promega, Madison WI) and oligo(dT) primers according to the manufacturer's instructions. Quantitative real time PCR (qRT-PCR) analysis was performed on $100 \mathrm{ng}$ of cDNA in a final volume of $20 \mu \mathrm{l}$ according to the FullVelocity SYBR Green instruction manual (Stratagene, La Jolla, CA) or with a home-made master mix containing GoTaq polymerase (Promega, WI) and its buffer, $0.2 \mathrm{mM}$ dNTPs, $2.5 \mathrm{mM} \mathrm{MgCl}_{2}$, ROX dye and SYBR green in a final volume of $20 \mu \mathrm{l}$. qRT-PCR was performed in an Mx3005P spectrofluorometric thermal cycler (Stratagene, La Jolla, CA). The data were calibrated to unwounded WT. Ubiquitinconjugating enzyme (UBC21) At5g25760 51 was used as reference gene. The thermal cycle conditions were: an initial denaturation at $95^{\circ} \mathrm{C}$ for $2 \mathrm{~min}$, followed by 40 cycles of $20 \mathrm{~s}$ at 95 ${ }^{\circ} \mathrm{C}, 30 \mathrm{~s}$ at $60{ }^{\circ} \mathrm{C}$ and $45 \mathrm{~s}$ at $72{ }^{\circ} \mathrm{C}$. Three or four biological replicates were used for each experiment. Primers used were:

\begin{tabular}{|c|c|c|c|}
\hline Locus & Gene & Forward primer & Reverse primer \\
\hline At5g25760 & UBC21 & CAGTCTGTGTGTAGAGCTATCATAGCAT & AGAAGATTCCCTGAGTCGCAGTT \\
\hline At5g13220 & JAZ10 & ATCCCGATTTCTCCGGTCCA & ACTTTCTCCTTGCGATGGGAAGA \\
\hline At5g24770 & $V S P 2$ & CCGTGTGCAAAGAGGCTTA & CACAACTTCCAACGGTCAC \\
\hline At2g17260 & GLR3.1 & GGCCAAGAATTCACCAGATGC & GACCAAGAATCGCGGTTGACA \\
\hline At4g35290 & GLR3.2 & ATTCACCAGAAGTGGCTGGG & TGAAGCTGTCCGGTTTCTGA \\
\hline At1g42540 & GLR3.3 & CGACCTTTCAACCGTCTTAT & TCGAGAAGCTAAACCAGAGAA \\
\hline
\end{tabular}




\begin{tabular}{|l|l|l|l|}
\hline At3g51480 & GLR3.6 & GATTAGAAGTGGGTTGGGGGA & GAGGCAATGGTGGAGGAAGT \\
\hline
\end{tabular}

\section{Transcriptomics}

Total RNAs from leaves were isolated and purified with RNeasy Plant Mini Kit (Qiagene, Germany). All RNA quantities were assessed with a NanoDrop®ND-1000 spectrophotometer and the RNA quality was assessed using RNA 6000 NanoChips with the Agilent 2100 Bioanalyzer (Agilent, Palo Alto, USA). For each sample, 300ng of total RNA were amplified using the MessageAmp ${ }^{\text {TM }}$ II-Biotin Enhanced Single Round aRNA Amplification Kit (AM1791, Ambion). $12.5 \mu \mathrm{g}$ of the resulting biotin-labelled cRNA was chemically fragmented. Affymetrix ATH1 (batch 1211501) arrays (Affymetrix, Santa Clara, CA, USA) were hybridized with $11 \mu \mathrm{g}$ of fragmented target, at $45^{\circ} \mathrm{C}$ for $17 \mathrm{~h}$ and washed and stained according to the protocol described in Affymetrix GeneChip ${ }^{\circledR}$ Expression Analysis Manual (Fluidics protocol FS450_0007). The arrays were scanned using the GeneChip ${ }^{\circledR}$ Scanner 3000 $7 \mathrm{G}$ (Affymetrix) and raw data was extracted from the scanned images and analyzed with the Affymetrix Power Tools software package (Affymetrix). Statistical analysis was performed using the free high-level interpreted statistical language $\mathrm{R}$ and various Bioconductor packages (http://www.Bioconductor.org). Hybridization quality was assessed using the Expression Console software (Affymetrix). Normalized expression signals were calculated from Affymetrix CEL files using the RMA normalization method. Differential hybridized features were identified using the Bioconductor package "limma" that implements linear models for microarray data ${ }^{52}$. The $P$ values were adjusted for multiple testing with Benjamini and Hochberg's method to control the false discovery rate (FDR) ${ }^{53}$. Probe sets showing at least 2fold change and a FDR $<0.05$ were considered significant. 


\section{JA and JA-Ile quantification}

Isopropanol and methanol were obtained from VWR Prolabo (Leuven, Belgium) and used for extraction analysis. Liquid chromatography mass spectrometry-grade acetonitrile and water from Biosolve (Dieuze, France) were used for the high-performance liquid chromatography. The internal standards used were $\left[{ }^{18} \mathrm{O}\right]_{2}$-jasmonic acid ${ }^{54}$ and $\left[{ }^{13} \mathrm{C}\right]_{6}$-jasmonoyl L-isoleucine ${ }^{55}$. Frozen leaves (200 mg, from 5 week-old plants) were ground in a ball mill extractor with internal standards (40 $\mathrm{ng} / \mathrm{ml})$ prior to extraction with isopropanol. Chlorophyll was removed with a $\mathrm{C} 18$ solid-phase extraction cartridge using $\mathrm{MeOH}: \mathrm{H}_{2} \mathrm{O}(85: 15, \mathrm{v} / \mathrm{v})$ for elution. The eluate was concentrated and dissolved in $100 \mu \mathrm{MeOH}: \mathrm{H}_{2} \mathrm{O}(85: 15$, v/v). Separation was carried out on a Phenomenex Kinetex (Torrance, CA) 2.6 mm C18 $100 \AA$ column $(100$ x 3.0 $\mathrm{mm}$ ). A gradient elutation was run at a flow rate of $0.4 \mathrm{~mL} / \mathrm{min}$ with the following solvent system: $\mathrm{A}=0.1 \%$ formic acid $/$ water, $\mathrm{B}=0.1 \%$ formic acid/ acetonitrile; $5 \% \mathrm{~B}$ for $3 \mathrm{~min}, 5$ $75 \% \mathrm{~B}$ in $11 \mathrm{~min}, 75-95 \% \mathrm{~B}$ in $2 \mathrm{~min}, 95 \% \mathrm{~B}$ for $2 \mathrm{~min}$ and $95-5 \% \mathrm{~B}$ in $2 \mathrm{~min}$. The electrospray ionisation conditions were as follows: capillary voltage $3300 \mathrm{~V}$; cone voltage 24 $\mathrm{V}$; extractor $3 \mathrm{~V}$; RF Lens $0 \mathrm{~V}$; source temperature $120^{\circ} \mathrm{C}$; desolvation temperature $350{ }^{\circ} \mathrm{C}$; cone gas flow $900 \mathrm{~L} / \mathrm{h}$ and desolvation gas flow $27 \mathrm{~L} / \mathrm{h}$. Jasmonates were monitored with quantitative multiple reaction monitoring $(\mathrm{MRM})$ in a Quattro micro ${ }^{\mathrm{TM}}$ API mass spectrometer (Waters, Milford, MA, USA) with an electrospray ionization interface coupled with the Agilent LC system (Hewlett Packard). Detection was performed in negative ion mode over an $\mathrm{m} / \mathrm{z}$ range of 100-1000. The MRM transitions were: JA: $209.1>58.7,{ }^{18} \mathrm{O}_{2} \mathrm{JA}$ : $213.1>62.8$, JA-Ile: $322.2>130.0$ and JA- $\left[{ }^{13} \mathrm{C}\right]_{6}$ Ile: $328.2>136.0$ (parent $>$ daughter). The limit of quantification (LOQ $=3 \mathrm{x}$ limit of detection) could reach up to $9.2 \mathrm{pmol} / \mathrm{g} \mathrm{FW}$ for JA and $4.5 \mathrm{pmol} / \mathrm{g} \mathrm{FW}$ for JA-Ile. Data below LOQ were considered as non-informative.

\section{Genotyping of T-DNA insertion lines}


T-DNA insertion lines were obtained from the Nottingham Arabidopsis Stock Centre (NASC) except for the respiratory burst oxidase homolog D mutant rbohD (Salk_070610) which was from Y. Lee (University of Lausanne, T-DNA line) and F. Mauch (University of Fribourg, $d S p m \operatorname{line}^{56}$ ). For genotyping, $5 \mathrm{mg}$ fresh leaf samples were placed into 96-well microtiter plates and tissues were ground using a Qiagen TissueLyser II (Retsch Technology $\mathrm{GmbH}$, Haan, Germany). Then, $60 \mu \mathrm{l}$ of extraction buffer $(200 \mathrm{mM}$ Tris $\mathrm{HCl} \mathrm{pH}$ 7.5, $250 \mathrm{mM}$ $\mathrm{NaCl}, 25 \mathrm{mM} \mathrm{Na} \mathrm{NDDT}_{2}$, $0.5 \%$ SDS) was added to each well and the samples were centrifuged at $14000 \mathrm{~g}$ for $10 \mathrm{~min}$. The supernatants were transferred into new microtiter plates and the same volume $(50 \mu \mathrm{l})$ of isopropanol was added. The plates were then centrifuged at $14000 \mathrm{~g}$ for $5 \mathrm{~min}$. The resultant pellets were washed with $70 \%$ ethanol (150 $\mu \mathrm{l})$ and centrifuged at $14000 \mathrm{~g}$ for $5 \mathrm{~min}$. Finally, DNA was resuspended in $50 \mu \mathrm{l}$ deionized water. $2 \mu 1$ of this extracted DNA was used as template for each final $20 \mu \mathrm{l}$ PCR reaction. The sequences of forward or reverse primers pairs used for genotyping are shown in Table S8.

\section{VSP2 $2_{\text {Pro }}:$ GUSPlus plant transformation}

The VSP2 (At5g24770) promoter, amplified using 5'-TTC TCT CTG GTT ATA TTT TGT TGC TG-3' and 5'-TGT TTA TAT GTG TGA CGC AAA GG -3' primers) was cloned with XmaI and KpnI (New England Biolabs, Ipswich, MA, USA) into the pUC57-L4-KpnI/XmaIR1 plasmid producing a pEN-L4-VSP2 ${ }_{P r o}-\mathrm{R} 1$ as an pENTRY clone. The pUC57-L4KpnI/XmaI-R1 plasmid was generated by Joop Vermeer (DBMV, University of Lausanne) by introducing L4-KpnI/XmaI-R1 att recombination and restriction sites into pUC57 (Invitrogen, Paisley, UK). pEN-L1-GUSPlus-L2 plasmids were obtained with Gateway technology according to manufacturer instructions (Invitrogen) with GUSPlus cDNA (amplified from

pCAMBIA1305.2 (CAMBIA, Australia) and pDONR $^{\mathrm{TM}} / \mathrm{ZEO}$ (Invitrogen). The final VSP $2_{\text {Pro }}:$ GUSPlus constructs were generated by using a double Gateway reaction into 
pEDO097pFR7m24GW. pEDO097pFR7m24GW was generated by inserting the FAST (fluorescence-accumulating seed technology) cassette $^{57}$ into $\mathrm{pH} 7 \mathrm{~m} 24 \mathrm{GW}$ (Invitrogen) by Ester M.N. Dohmann (DBMV, University of Lausanne). WT plants were transformed using Agrobacterium tumefaciens cells as described by Berberich et al. ${ }^{58}$. Transformed seeds expressing red fluorescence protein (RFP) were selected by florescence microscopy. The $T_{1}$ generation was used for experiments.

\section{Supplementary References:}

48 van Wees, S. Phenotypic Analysis of Arabidopsis Mutants: Trypan Blue Stain for Fungi, Oomycetes, and Dead Plant Cells. Cold Spring Harb Protoc. doi:10.1101/pdb.prot4982 (2008).

49 Jefferson, R. A., Kavanagh, T. A. \& Bevan, M. W. GUS fusions: beta-glucuronidase as a sensitive and versatile gene fusion marker in higher plants. $E M B O J . \mathbf{2 0}, 3901$ 3907 (1987).

50 Onate-Sanchez, L. \& Vicente-Carbajosa, J. DNA-free RNA isolation protocols for Arabidopsis thaliana, including seeds and siliques. BMC Res Notes 1, 93-100 (2008).

51 Czechowski, T., Stitt, M., Altmann, T., Udvardi, M. K. \& Scheible, W.-R. GenomeWide Identification and Testing of Superior Reference Genes for Transcript Normalization in Arabidopsis. Plant Physiol. 139, 5-17 (2005).

52 Smyth Gordon, K. Linear Models and Empirical Bayes Methods for Assessing Differential Expression in Microarray Experiments. Stat. Appl. Genet. Mol. Biol. 3 (2004).

53 Benjamini, Y. \& Hochberg, Y. Controlling the False Discovery Rate: A Practical and Powerful Approach to Multiple Testing. J. R. Stat. Soc. Ser. B Methodol. 57, 289-300 (1995).

54 Mueller, M. J., Mène-Saffrané, L., Grun, C., Karg, K. \& Farmer, E. E. Oxylipin analysis methods. Plant J. 45, 472-489 (2006).

55 Kramell, R., Schneider, G. \& Miersch, O. Chiral separation of amide conjugates of jasmonic acid by liquid chromatography. Chromatographia 45, 104-108 (1997).

56 Torres, M.A., Dangl, J.L. \& Jones JD. Arabidopsis gp91phox homologues AtrbohD and AtrbohF are required for accumulation of reactive oxygen intermediates in the plant defense response. Proc. Natl. Acad. Sci U S A 99, 517-522 (2002)

57 Shimada, T. L., Shimada, T. \& Hara-Nishimura, I. A rapid and non-destructive screenable marker, FAST, for identifying transformed seeds of Arabidopsis thaliana. Plant J. 61, 519-528 (2010).

58 Berberich, T., Takahashi, Y., Saitoh, H. \& Terauchi, R. in The Handbook of Plant Functional Genomics 113-136 (Wiley-VCH Verlag GmbH \& Co. KGaA, 2008). 


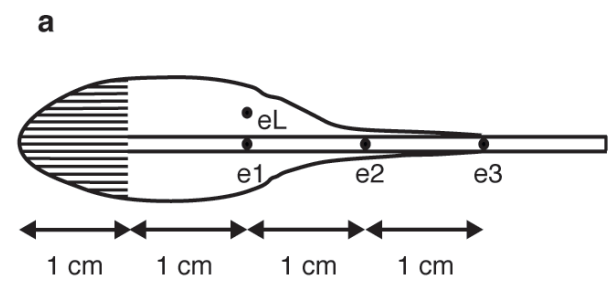

e
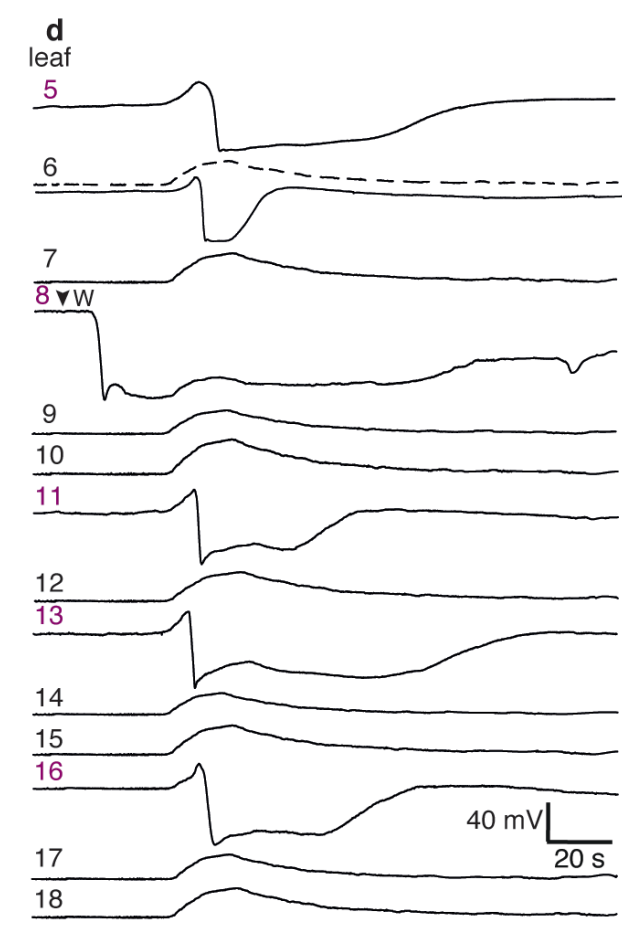

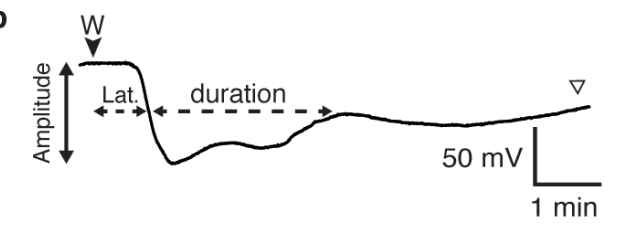

b

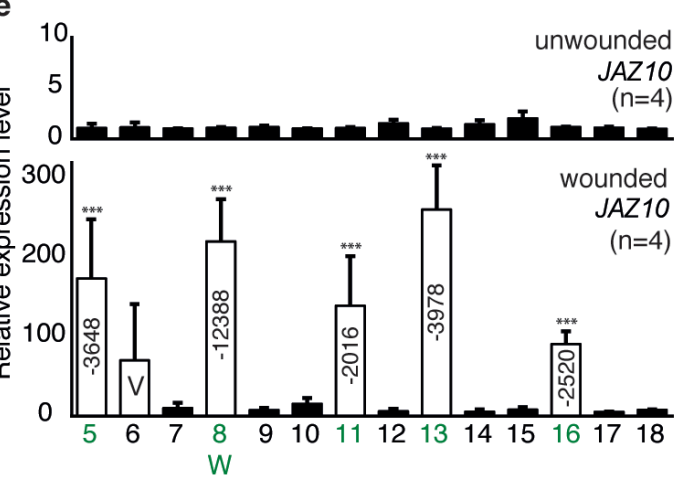

\section{f}

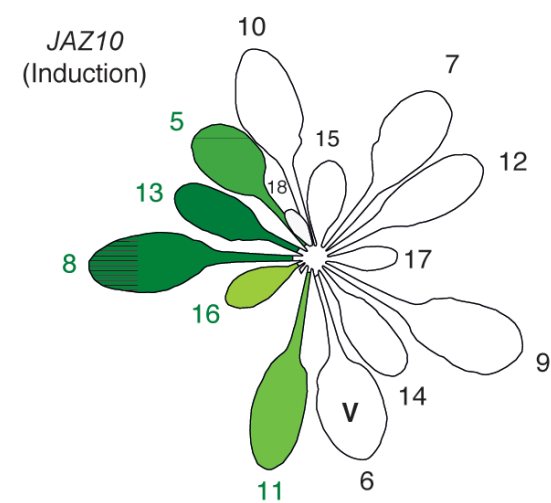

C

Untouched

$\checkmark$ Touched

eL $\checkmark$ Wounded
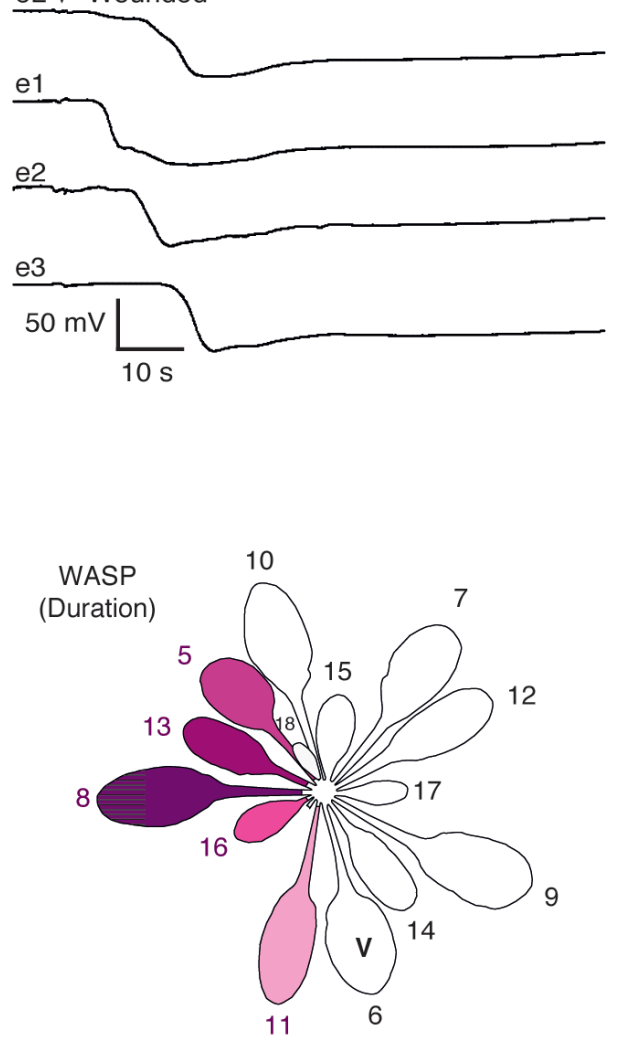

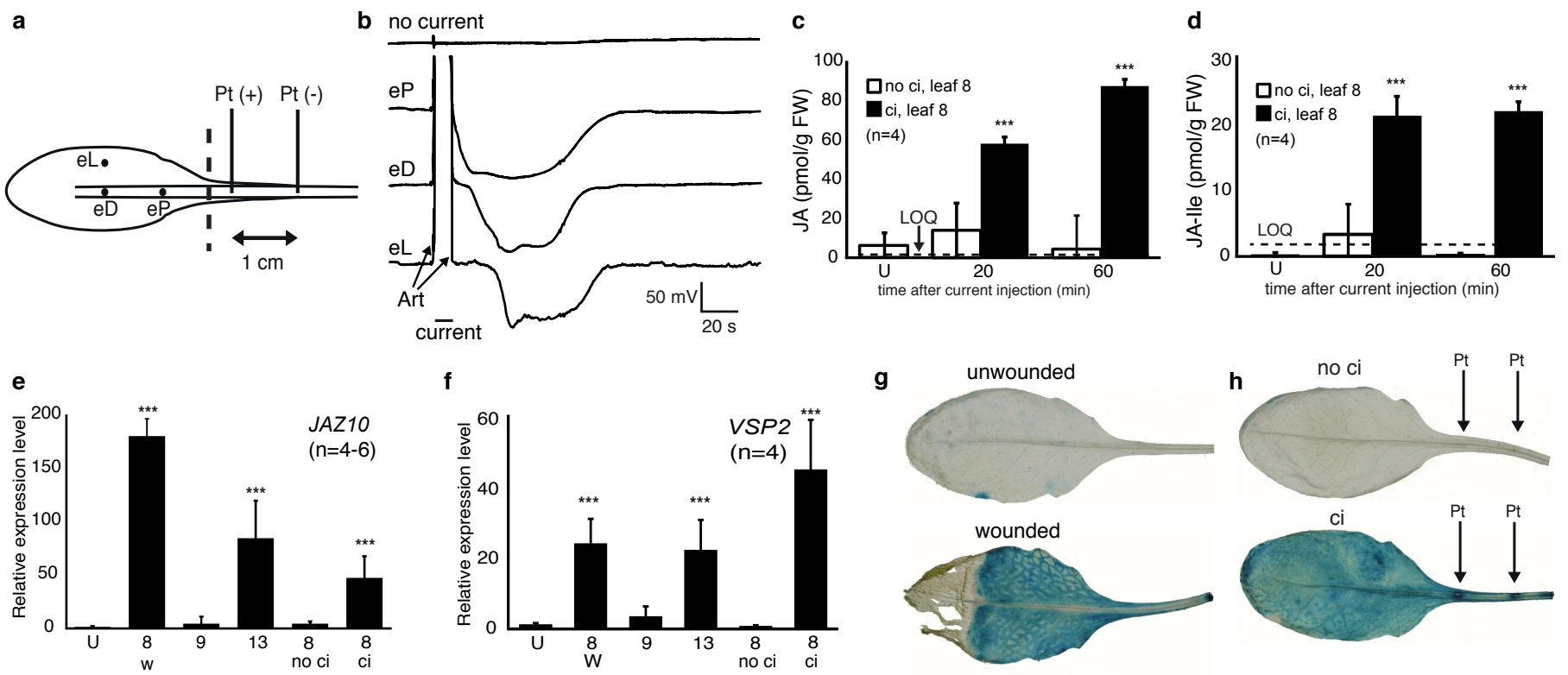


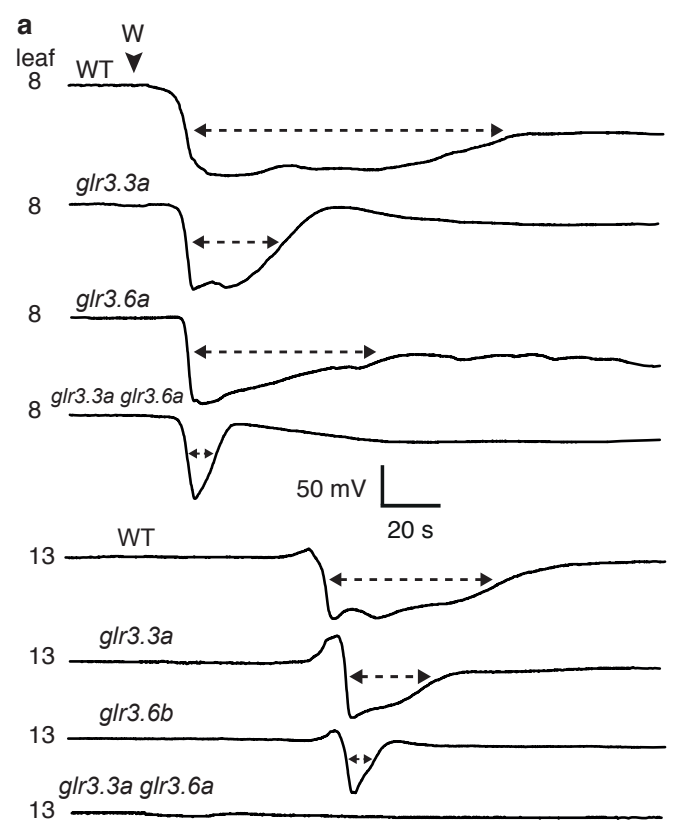

b

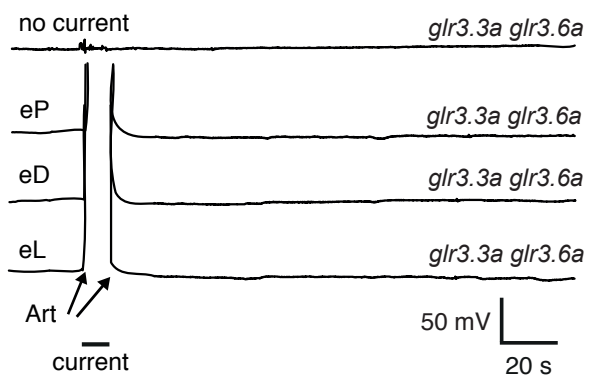

d

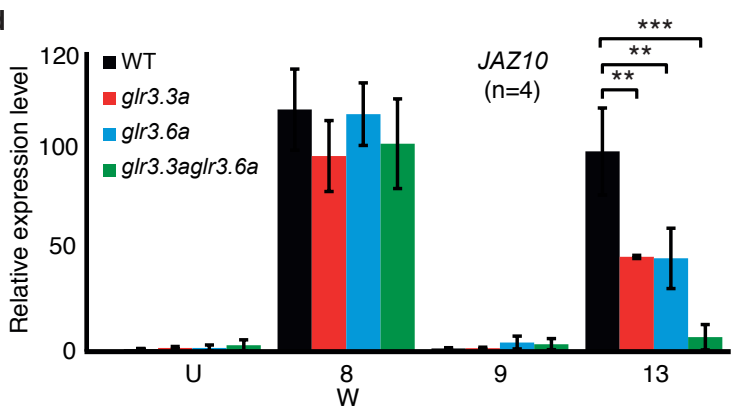

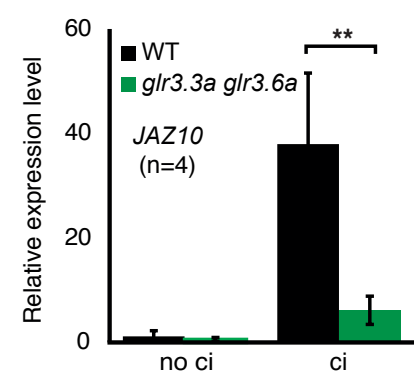

